\title{
'CHASING OFF THE DEVIL' : MEDICO-LEGAL ISSUES RELATED TO INDIGENOUS TREATMENT OF A CHILD.
}

\author{
Senanayake S M H M K \\ Judicial Medical Officer's Office, Teaching Hospital Kurunegala, Sri Lanka
}

\begin{abstract}
Indigenous remedies are often used as treatment for mental illnesses in Sri Lanka. This communication reports a case of a girl who underwent burn injuries to soles as a part of indigenous treatment.
\end{abstract}

A 14-year-old girl who developed sudden convulsions had her soles burnt by iron rods by an indigenous healer as a treatment. She was later admitted to a general hospital and treated for burn injuries on both soles and dissociative convulsions. She had to undergo skin graft, had a prolonged hospital stay, and was compelled to wear protective footwear for the rest of her life. Her burn injuries were initially categorized as firstgrade, and non-grievous, by a medico-legal practitioner. However, a second medicolegal examination done under magistrate order by a Consultant Judicial Medical officer classified the injury as grievous as she had deep burns and a two-month hospital stay. Injuries were compatible with the history.

Review of the injury is necessary in burns of soles and palms due to the difficulty of recognizing the depth of the burn on initial examination. Request for a second medicolegal examination by a more competent medico-legal specialist or by a board of specialists is an alternative strategy available for victims and judiciary when the first medico-legal report is unsatisfactory.

Key words : Indigenous treatment, burns, medico-legal issues

\section{INTRODUCTION}

Indigenous remedies are often used as treatment for mental illnesses in Sri Lanka. They are typically done at places termed as 'Devalayas' which are places of worship of Deities. This report is about a girl who underwent burn injuries to soles as a part of indigenous treatment.

\section{Case history}

A 14 year old girl developed sudden unconsciousness at home. She was taken by family members to a nearby 'Devalaya'. This particular Devalaya was run by an English school master, and it was believed that, when put into a trance, a god will take possession of his body. Upon seeing the subject, the school master had claimed that she was, in fact, 'possessed by the devil', and had gone on to burn the girl's soles with an iron rod to chase off the devil. He had reassured her family that she will not come to any harm. The girl did not regain consciousness after having her soles burnt. Since this supposed remedy did not work she was admitted to a nearby general hospital. She was treated for burn injuries of both soles and dissociative convulsions.

Medico-legal practitioner found burn injuries on both soles which were initially assumed to be first degree burns and categorized as nongrievous. The schoolmaster was arrested for physical child abuse and then released after discovering that harm is due to a form of treatments which is culturally accepted in the local area. The girl needed skin grafts for both feet and protective sole wears for rest of life to protect the grafted skin taken from thighs. 
A second Medico-legal examination done under magistrate order by specialist forensic medicine practitioner stated that the injuries were grievous because of the two month stay in hospital due to deep burns and skin graft.

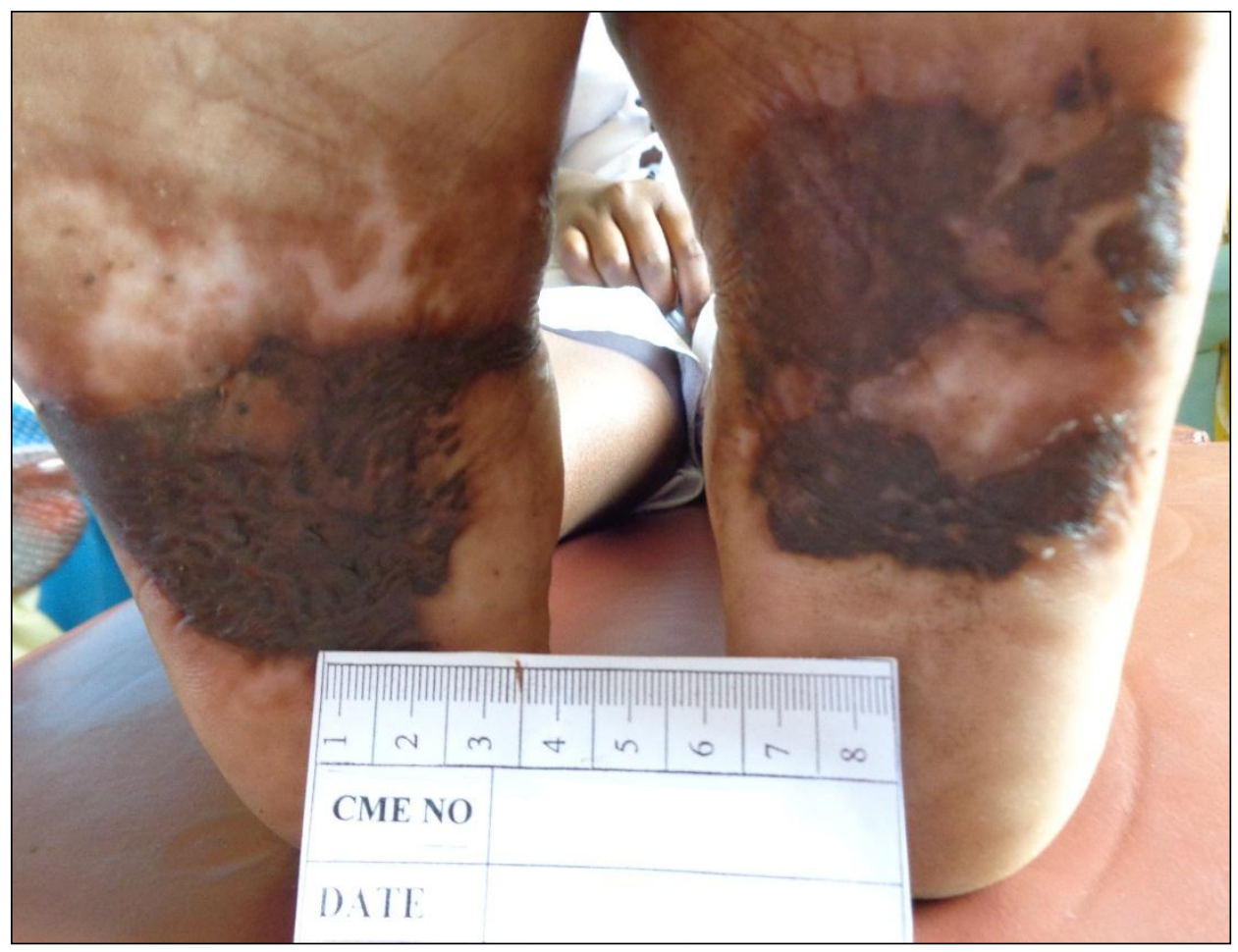

Figure 1 : Deep burns before skin graft

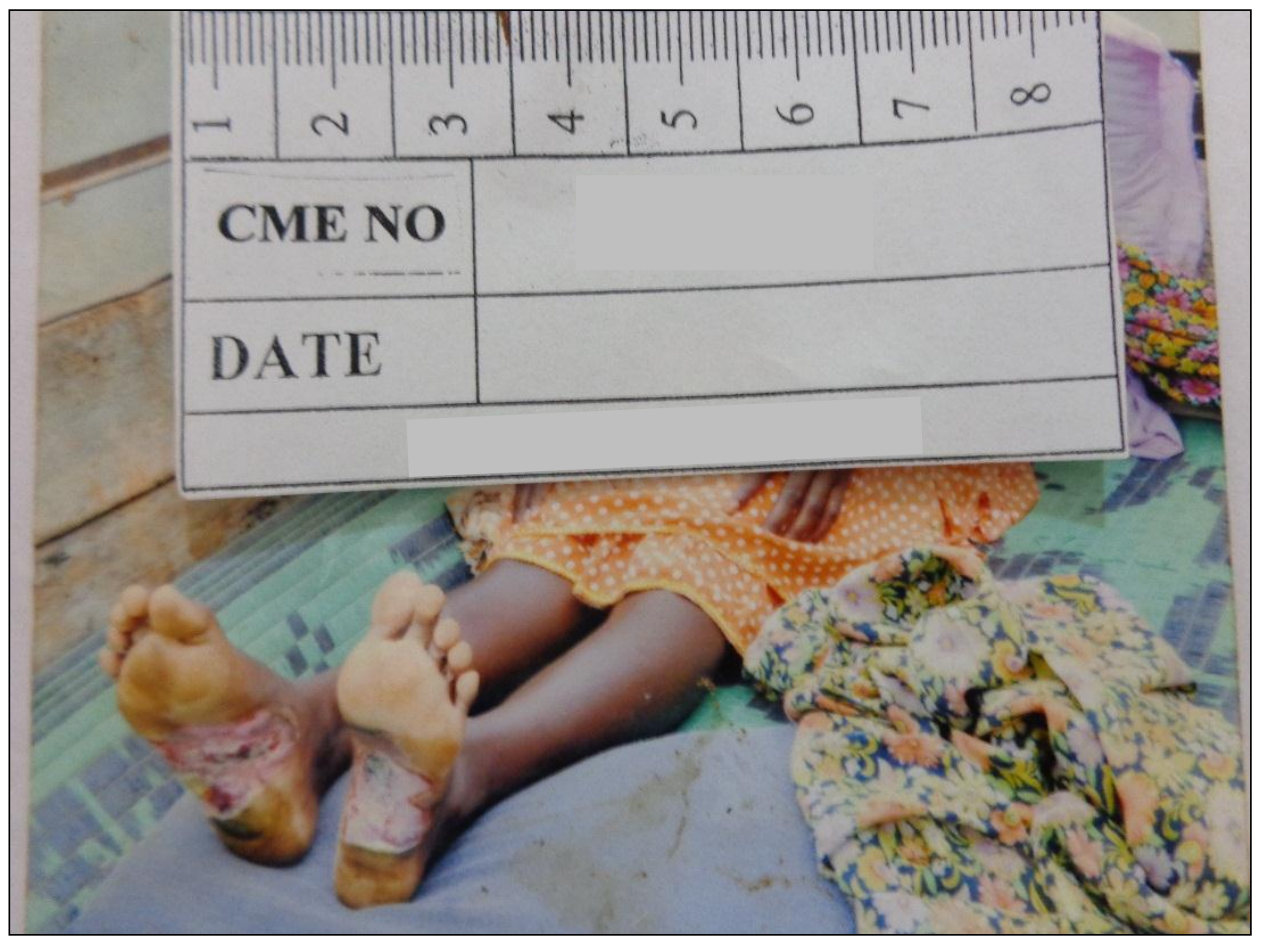

Figure 2 : Healed burns after skin graft

Sri Lanka Journal of Forensic Medicine, Science \& Law-May 2015-Vol.6 No.1 


\section{DISCUSSION}

Burn injuries in the soles could be accidental, self-inflicted, or intentional. They can also occur due to prolonged walking with bare feet $^{1}$. The depth of the burn varies according to the temperature, duration of the exposure and nature of the skin. The size of the burn, depth of burn, injury pattern and complications are relevant for the forensic medicine practitioner in order to address relevant medico legal issues.

The assailant in this case is a school master as well as an indigenous healer who treats patients probably under what is claimed to be a possession trance in his temple. A possession trance is characterized by a supposed transient replacement of a person by a spirit, ghost, deity, or other person ${ }^{2}$. The experience of being "possessed" by another entity, such as a person, god, demon, animal, or inanimate object, holds different meanings in different cultures. Lay people believe such healers have supernatural powers ${ }^{3}$. Therefore some healers 'pretend' to be in a trance in order to treat others, especially those with minor mental illnesses.

The following medico-legal issues were found in this case.

The depth of burn injuries of palms and soles is difficult to distinguish as first degree, second degree or third degree due to the thickness of the skin. Review of the patient after a few days is necessary because when a burn is examined on the first few days all three zones $^{5}$ (zone of coagulation, zone of stasis and zone of hyperemia) of a burn injury will be seen as one patch with inflammation. After few days sloughing will take place and the real depth will be evident. In this case the first medicolegal practitioner recognized burns as first degree burns and medicolegally categorized it as a non-grievous injury. The second examiner who examined the patient after skin grafting categorized the burn injury as grievous injury because of "unable to follow ordinary pursuits for a period of 20 days". 6

1. The conclusions of the second medicolegal report differed from the first. If any interested party is not happy with a medico-legal report in the court of law as in this case, a second Medico-Legal examination can be ordered by magistrate. In a complex case even a medical board can be appointed by the court.

2. Pattern of burn injury was important to determine the compatibility with the clinical history. In this case burns were not confined only to weight bearing sites of soles but involved the center of feet which are elevated. Therefore this injury was not due to an accidental walk on fire but more likely due to an intentional direct contact with a heated object as mentioned in the clinical history. Contact burns are usually seen in long duration barefoot walking, epilepsy, unconscious people and alcohol and drug abusers ${ }^{4}$.

3. Mild form of mental illnesses are treated by indigenous healers using various methods like beating, burning, chanting, dancing and drumming. There should be some form of mechanism to control harmful treatment / methods by unregistered healers, under possession trance or pretended possession trance. In this case action under criminal law was not taken because it was a form of treatment provided with the consent of the subject's mother.

4. Civil compensation case had been filed and sufficient amount of money was requested for the suffering of patient over several months, expenses for the treatments and skin graft, expenses for the protective soles which patient is supposed to wear throughout life to prevent injuries of grafted skin on soles. 


\section{RECOMMENDATIONS}

Review of the injury is necessary in burns of soles and palms due to the difficulty of recognizing the depth of the burn for categorizing the harm. Request for a second medico-legal examination by a more competent medico-legal specialist or by a board of specialists is an alternative strategy available for victims and judiciary when the first medico-legal report is unsatisfactory or disputed.

\section{REFERENCES}

1. Shakirow BM., Tursunov BS. Treatment of severe foot burns in children. Burn. 2005 Nov; 11(7) 901-9052.

2. Wijesinghe CP., Dissanayake SAW., Mendis N. Possession Trance in a Semi-Urban Community in Sri Lanka. Australian and New Zealand Journal of psychiatry. 1976, Vol. 10, No. 2: 135-139.

3. Ee Heok Kua, Li Ping Sim, Kuan Tsee Chee. A Cross-Cultural Study of the PossessionTrance in Singapore. Australian and New Zealand Journal of psychiatry. September 1986; Vol 20 No. 3: 361-364.

4. Abu Sittar GA \& et al. Thermal injury to hand: Review of literature. Annal's of Burns and Fire disasters. 2011 Dec 31; 24(4): 175185.

5. Hettirachchi S, Dziewulski P. Pathophysiology and types of burns, BMJ 2004 June 12; 328(7453): 1427-1429.

6. Srilankan Penal Code, Section 311; limbi. 